\title{
Developing family-based care for schizophrenia: a training project
}

\author{
Ian R. H. Falloon, Consultant Physician in Mental Health; Victor Hole, \\ Senior Nurse Therapist in Behavioural Psychotherapy; William ShanaHaN, \\ Senior Registrar, MARC LAPORTA, Research Associate*; and HAROUTYON KREKORIAN, \\ Research Director, Buckingham Mental Health Service, 22 High Street, Buckingham \\ MK18 1NU
}

In recognition of the fact that schizophrenia disorders remain a major public health problem presenting a severe limitation to the success of community-based approaches to mental health care, a programme was started in 1985 with the goal of developing family care of schizophrenia within the NHS.

The Family Care Training for Schizophrenia, or FACTS Project as it is known, was established against a background of information relating to the pharmacotherapy and psychological treatments of schizophrenia and the training of relevant personnel.

It was noted that despite optimal pharmacotherapy, the risk of schizophrenic illness following a chronic course remains high, with half the cases suffering persistent symptoms, recurrent episodes, or serious reactions to their drugs, during the first year after an episode (Carpenter \& Schooler, 1981). However, over the past decade a series of controlled studies has demonstrated the benefits of continuing optimal drug therapy with psychological treatments that focus on the management of environmental stress factors (Kuipers \& Bebbington, 1988). Most of these approaches have included key caregivers involved in the training of stress-management techniques. In addition to achieving significant reductions in clinical morbidity, improved quality of life for both patients and caregivers has been evident. Furthermore, the reduced costs for intensive care exceed the added costs of providing home based treatment, making the approach highly efficient (Falloon, 1985). The FACTS Project seeks to exploit this evidence, the strength of which makes it difficult to justify the current lack of provision of such services throughout the NHS.

The FACTS Project also redresses another fundamental imbalance. One major reason for delays in developing psychosocial treatments for schizophrenia has been the lack of training facilities. The Programme provides these. Another crucial deficit is the lack of multidisciplinary community-based teams

*Sponsored by a grant from the Fonds de Recherche en 'Sante du Quebec, Montreal, Quebec, Canada. that can readily continue optimal drug therapy with psychosocial interventions.

Psychiatrists, nurses, social workers, occupational therapists and psychologists all have vital roles to play in the effective case management of schizophrenia. Competent teamwork is a basic ingredient of all successful programmes. The FACTS Project recognises these parameters.

Following a pilot project in Southampton (Whitfield et al, 1987) funding was received from the Gatsby Trust and NUPRD and the efforts of the programme were extended. This development programme assists health districts to develop multidisciplinary teams and to train their staff in the skills of family-based stress management. Over 200 hours of consultation, training and supervision are provided over a 12-month period. This is conducted in four phases.

Phase One involves consultation with senior clinicians and managers about the planning and implementation of the multidisciplinary development. At least one on-site visit is needed, usually including a lecture on the benefits and costs of the programme, and meetings with all key unit personnel. Two experienced clinicians are chosen who have expressed an interest in co-directing the project and have good teaching skills.

In Phase Two, the two clinicians are trained not only in the treatment methods, but also in the skills needed to train and supervise other clinicians in this approach. The trainers complete a series of reading assignments using manuals and workbooks on the approach. They also listen to audiotaped materials on the diagnosis and the patients' and families' experiences of schizophrenia. After this preparation the two project directors/trainers attend a three-day intensive workshop where they learn the basic skills of family management, as well as specific peer supervision methods.

The approach is based upon widely utilised methods of behavioural family therapy, but includes education about the nature of schizophrenia and its comprehensive management, recognising the early 
warning signs of recurrent episodes and devising strategies to deal with specific difficulties that may arise, e.g. suicidal behaviour, social skills deficits, amotivational states (Falloon et al, 1984). The aim is not merely to reduce all forms of stress that impinge on the patient, but at the same time to promote the quality of life of the patient and his caregivers.

The major therapeutic strategy involves training families to solve problems in a highly efficient manner. After detailed assessment of the problem solving skills of the family unit, functional deficits are corrected so that all household members can communicate their needs and concerns and resolve them quickly and constructively. In addition to training in a structured approach to problem solving, families may be taught specific communication skills, such as making requests, or listening attentively. The therapist avoids solving problems for families, and focuses on teaching strategies for resolving their own problems. This is facilitated by assigning homework for the family to practise the skills taught in the sessions.

The straightforward structure of the approach enables families to acquire the skills readily, and therapists to train others in the methods without difficulty. The training workshops employ identical training methods to those used with families. Each training session is introduced with a brief rationale for the skill that is being taught; a demonstration of the skill is provided (usually on a videotape); this is followed by practice of the skill with a roleplayed family. Constructive feedback is provided until the clinician performs the skill competently.

During Phase Three tutoring is provided over a six-month period during which the project directors audiotape at least 30 sessions of two families. These sessions are reviewed by FACTS supervisors who provide immediate feedback. Therapist competence is assessed on a session to session basis using a standardised rating scale.

On completion of the co-directors' training, a further three-day workshop is conducted on-site for a further 12 clinicians. The two co-directors assist in leading the workshop along with a FACTS supervisor. Additional meetings with management staff may be held during this on-site visit to iron out any difficulties that have not been resolved by the codirectors. An additional half-day workshop may be conducted to train physicians in the latest developments in drug management of schizophrenia (Carpenter \& Schooler, 1983).

In the final part of Phase Four the clinicians are supervised on the treatment of two families (30 sessions over six months) by the co-directors/ trainers. This is usually conducted in a local supervision group. Additional consultations with the FACTS supervisors provide further support to the developing service. A one-day refresher workshop may be arranged in the second year of the project.

By the end of 1988, 28 co-directors/trainers had completed their initial workshop training, a further 33 are booked for the first half of 1989. It is hoped that within another two years sufficient skilled trainers will be available to develop similar programmes in all health districts. It is anticipated that within five years every person suffering from schizophrenia in Britain will be able to receive an optimal combination of drug and psychosocial therapy.

\section{References}

CARpenter, W. T. \& SchOOLer, N. R. (1983) New directions in drug treatment for schizophrenia. Schizophrenia Bulletin, 9, 1-63.

FALLOON, I. R. H. (1985) Family Management of Schizophrenia: A Controlled Study of Clinical, Social, Family and Economic Benefits. Baltimore: Johns Hopkins University Press.

-, BoYD, J. L. \& MCGill, C. W. (1984) Family Care of Schizophrenia. New York: Guilford Press.

KUIPERS, L. \& BEBBINGTON, P. (1988) Expressed emotion research in schizophrenia: theoretical and clinical implications. Psychological Medicine, 18, 893-910.

WhITFIELD, W., TAYLOR, C. \& VIRGO, N. (1988) Family care in schizophrenia. Journal of the Royal Society of Health. $1,3-5$. 